\title{
A GENERALIZED THERMOELASTIC DUAL-PHASE-LAGGING RESPONSE OF THICK BEAMS SUBJECTED TO HARMONICALLY VARYING HEAT AND PRESSURE
}

\author{
Ashraf M. Zenkour \\ Department of Mathematics, Faculty of Science, King Abdulaziz University, Jeddah, Saudi Arabia and \\ Department of Mathematics, Faculty of Science, Kafrelsheikh University, Kafreelsheikh, Egypt \\ e-mail:zenkour@kau.edu.sa; zenkour@sci.kfs.edu.eg
}

\begin{abstract}
The generalized thermoelastic problem of a thermo-mechanically loaded beam is studied. The upper surface of the beam is thermally isolated and subjected to a mechanical load while the bottom surface is traction free and subjected to a heating source. Based on the heat conduction equation containing the thermoelastic coupling term and the two-dimensional elasticity theory, thermoelastic coupling differential equations of motion are established. The generalized thermoelasticity theory with the dual-phase-laggings (DPLs) model is used to solve this problem. A closed-form analytical technique is used to calculate vibration of displacements and temperature. The effects of the phase-laggings (PLs), the intensity of the applied load and heat parameters on the field quantities of the beam are discussed. The variation along the axial direction and through-the-thickness distributions of all fields are investigated. Some comparisons have been also shown graphically to estimate the effects of the time on all the studied fields.
\end{abstract}

Keywords: thermoelasticity, dual-phase-lag model, two-dimensional elasticity solution

\section{Nomenclature}

$C^{e} \quad-\quad$ specific heat per unit mass at constant strain

e $\quad-$ volumetric strain

E $\quad-$ Young's modulus

$K \quad-$ thermal conductivity

$L, h \quad-\quad$ beam length and thickness, respectively

$Q^{*} \quad-$ heat source

q $\quad-$ heat flux vector

$T(x, z, t) \quad$ - temperature distribution

$T_{0} \quad-$ environmental temperature

u $\quad-$ displacement vector

$u, w \quad-\quad$ axial and transverse displacements

$u^{*}, w^{*} \quad-\quad$ amplitudes of axial and transverse displacements

Greek symbols

$\alpha \quad-$ linear thermal expansion coefficient

$\delta \quad-\quad$ unification parameter

$\varepsilon_{i j}, \sigma_{i j} \quad-$ strain and stress tensor, respectively

$\gamma \quad-$ stress-temperature modulus, $\gamma=E \alpha /(1-2 \nu)$

$\lambda^{*}, \mu^{*} \quad-\quad$ Lamé's constants

$\nu \quad-$ Poisson's ratio

$\omega \quad-\quad$ angular frequency

$\rho \quad-\quad$ material density of medium 


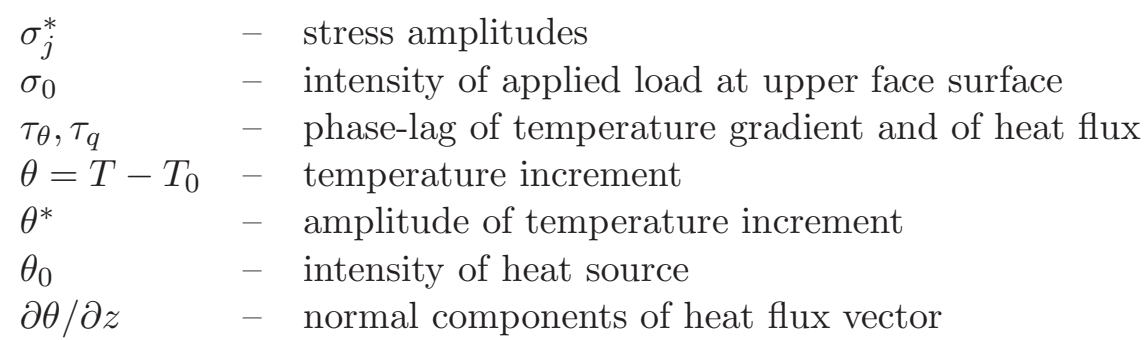

\section{Introduction}

Many generalized theories of thermoelasticity have been developed in the literature to study the behavior of thermoelastic structures. These theories can be classified in different models, such as the theory of coupled thermoelasticity (CTE) (Biot, 1956), the Lord and Shulman (L-S) theory (Lord and Shulman, 1967), the Green and Lindsay (G-L) theory (Green and Lindsay, 1972), the Green and Naghdi (G-N) theory (Green and Naghdi, 1991, 1992, 1993) as well as the Tzou (1955a,b, 1006) dual-phase-lag (DPL) thermoelasticity theory (see also Chandrasekharaia (1998)). To the author's best knowledge, only a few authors have presented the exact two-dimensional solution to the generalized thermoelastic beam problem up to present time. Most authors used the classical theory for thin beams as well as one of the generalized thermoelasticity theories.

The investigation of harmonic plane wave propagations in an elastic medium have been attempted by several researchers. Prasad et al. (2010) investigated the propagation of harmonic plane waves with an assigned frequency by employing the thermoelasticity theory with dual-phase-lags. Mukhopadhyay (2004) presented thermoelastic interactions without energy dissipation in a spherical-cavity medium subjected to harmonically varying temperature. Kobzar' and Fil'shtinskii (2008) presented the plane dynamic problem of coupled thermoelasticity takeing into account the harmonic form of the change of field quantities with time. Allam et al. (2009) presented the 2-D problem of electromagneto-thermoelasticity for a perfectly conducting thick plate subjected to a harmonically time-dependent heat source in the context of G-N theory. Ram et al. (2008) obtained a general solution to the field equations of a harmonically timedependent generalized thermodiffusion in an elastic solid. Mukhopadhyay and Kumar (2008) studied thermoelastic interactions in a spherical-cavity medium subjected to a time-dependentheating effect in the context of different thermoelasticity theories. Gue et al. (2012) analyzed thermoelastic damping of a micro-beam resonator by the dual-phase-lag thermal conduction model of the generalized thermoelasticity theory. Zenkour and Abouelregal (2014) presented nonlocal thermoelastic vibrations for variable thermal conductivity nanobeams due to harmonically varying heat. Recently, Zenkour (2015) presented a three-dimensional thermal shock plate problem within the framework of different thermoelasticity theories.

The present article is concerned with the two-dimensional transient generalized thermoelastic problem for a thick beam subjected to thermal and thermomechanical loads at its faces. Based on the dual-phase-lags model (Abouelregal and Zenkour, 2014; Abbas and Zenkour, 2014; Zenkour and Abouelregal, 2015; Zenkour et al., 2013), the exact closed-form solution for the governing equations is established. The equations of the classical thermoelasticity theory, Lord and Shulman theory, and Green and Naghdi theory may be established as special cases of the DPLs theory. All expressions for temperature, displacements and stresses are presented. Numerical results showing the thermoelastic dynamic responses of the field quantities through the axial and thickness directions of the beam are presented. The effect of the time parameter is also investigated. 


\section{Thermoelastic basic equations}

Let us consider a homogenous isotropic thermoelastic solid in the Cartesian coordinate system Oxyz initially un-deformed and at a uniform temperature $T_{0}$. The basic governing equations of motion, balance of the equilibrated force and heat conduction in the context of generalized (non-Fourier) thermoelasticity for the displacement vector $\mathbf{u}(x, y, z, t)$ in the absence of body forces should be considered.

The modified classical thermoelasticity model is given by the Tzou theory in which the Fourier law is replaced by an approximation of the equation

$$
\mathbf{q}\left(x, t+\tau_{q}\right)=-K \nabla T\left(x, t+\tau_{\theta}\right)
$$

The above equation may be approximated by

$$
\left(1+\tau_{q} \frac{\partial}{\partial t}\right) \mathbf{q}=-K\left(1+\tau_{\theta} \frac{\partial}{\partial t}\right) \nabla T
$$

where $0<\tau_{\theta} \leqslant \tau_{q}$. Then the heat conduction equation corresponding to the dual-phase-lag model proposed by Tzou in this case takes the form

$$
K\left(1+\tau_{\theta} \frac{\partial}{\partial t}\right) \nabla^{2} \theta+\left(1+\tau_{q} \frac{\partial}{\partial t}\right) \rho Q^{*}=\left(\delta+\tau_{q} \frac{\partial}{\partial t}\right)\left(\rho C^{e} \frac{\partial \theta}{\partial t}+\gamma T_{0} \frac{\partial e}{\partial t}\right)
$$

Equation (2.3) describes the coupled dynamical thermoelasticity theory (CTE), the generalized thermoelasticity theories proposed by Lord and Shulman (L-S), Green and Naghdi (G-N) theory and dual-phase-lag (DPL) model for different sets of values of phase-lags parameters $\tau_{q}$, $\tau_{\theta}$ and the unification parameter $\delta$ as follows:

CTE: $\tau_{\theta}=\tau_{q}=0$ and $\delta=1$

L-S: $\quad \tau_{\theta}=0, \tau_{q}=\tau_{0}\left(\tau_{0}\right.$ is the relaxation time $)$ and $\delta=1$

G-N: $\tau_{\theta}=0, \delta=0, \tau_{q} \neq 0$, and $K=K^{*}$ (the material constant characteristic)

DPL: $\quad \delta=1$ and $0<\tau_{\theta} \leqslant \tau_{q}$

\section{Governing equations}

Let us consider small flexural deflections of an elastic beam with dimensions $(L \times b \times h)$ as shown in Fig. 1. The beam may be subjected to various thermal and mechanical loads according to the type of the problem used. Let $u, v=0$ and $w$ denote displacement components of a material point located at $(x, y, z)$ in the present beam in the $x, y$, and $z$ directions, respectively. The stress-strain relationships in the beam coordinates are written in the form

$$
\left\{\begin{array}{l}
\sigma_{x} \\
\sigma_{z}
\end{array}\right\}=\frac{E}{(1+\nu)(1-2 \nu)}\left[\begin{array}{cc}
1-\nu & \nu \\
\nu & 1-\nu
\end{array}\right]\left\{\begin{array}{l}
\varepsilon_{x}-\alpha \theta \\
\varepsilon_{z}-\alpha \theta
\end{array}\right\} \quad \sigma_{x z}=\frac{E}{2(1+\nu)} \varepsilon_{x z}
$$

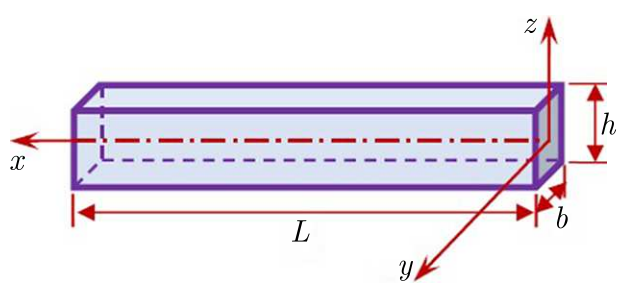

Fig. 1. Schematic diagram of the beam 
The strain-displacement relations are taken in the linear form

$$
\varepsilon_{x}=\frac{\partial u}{\partial x} \quad \varepsilon_{z}=\frac{\partial w}{\partial z} \quad \varepsilon_{x z}=\frac{\partial w}{\partial x}+\frac{\partial u}{\partial z}
$$

The governing equations of motion $\sigma_{i j, j}=\rho \ddot{u}_{i}$ can be presented in an expanded form as

$$
\begin{aligned}
& \frac{E(1-\nu)}{(1+\nu)(1-2 \nu)} \frac{\partial^{2} u}{\partial x^{2}}+\frac{E}{2(1+\nu)} \frac{\partial^{2} u}{\partial z^{2}}+\frac{E}{2(1+\nu)(1-2 \nu)} \frac{\partial^{2} w}{\partial x \partial z}-\frac{E \alpha)}{(1+\nu)(1-2 \nu)} \frac{\partial \theta}{\partial x}=\rho \frac{\partial^{2} u}{\partial t^{2}} \\
& \frac{E}{2(1+\nu)(1-2 \nu)} \frac{\partial^{2} u}{\partial x \partial z}+\frac{E}{2(1+\nu)} \frac{\partial^{2} w}{\partial x^{2}}+\frac{E(1-\nu)}{(1+\nu)(1-2 \nu)} \frac{\partial^{2} w}{\partial z^{2}}-\frac{E \alpha)}{(1+\nu)(1-2 \nu)} \frac{\partial \theta}{\partial z}=\rho \frac{\partial^{2} w}{\partial t^{2}}
\end{aligned}
$$

In addition, the thermal conduction equation for the beam without a heat source $\left(Q^{*}=0\right)$ is given as

$$
\left(1+\tau_{\theta} \frac{\partial}{\partial t}\right)\left(\frac{\partial^{2} \theta}{\partial x^{2}}+\frac{\partial^{2} \theta}{\partial z^{2}}\right)=\left(\delta+\tau_{q} \frac{\partial}{\partial t}\right)\left[\eta \frac{\partial \theta}{\partial t}+\frac{\gamma T_{0}}{K} \frac{\partial}{\partial t}\left(\frac{\partial u}{\partial x}+\frac{\partial w}{\partial z}\right)\right]
$$

where $\eta=\rho C^{e} / K$. Now, the following dimensionless definitions will be used for the variable quantities

$$
\begin{aligned}
& \{\bar{x}, \bar{z}, \bar{L}, \bar{h}, \bar{u}, \bar{w}\}=\eta c\{x, z, L, h, u, w\} \quad\left\{\bar{t}, \bar{\tau}_{0}, \bar{\tau}_{\theta}, \bar{\tau}_{q}\right\}=\eta c^{2}\left\{t, \tau_{0}, \tau_{\theta}, \tau_{q}\right\} \\
& \bar{\theta}=\frac{\theta}{T_{0}} \quad\left\{\bar{\sigma}_{x}, \bar{\sigma}_{z}\right\}=\frac{(1+\nu)(1-2 \nu)}{E}\left\{\sigma_{x}, \sigma_{z}\right\} \\
& \bar{\sigma}_{x z}=\frac{2(1+\nu)}{E} \sigma_{x z} \quad c^{2}=\frac{E}{\rho(1+\nu)(1-2 \nu)}
\end{aligned}
$$

Therefore, the heat equation and equations of motion are given by (dropping the prime for convenience)

$$
\begin{aligned}
& \left(1+\tau_{\theta} \frac{\partial}{\partial t}\right)\left(\frac{\partial^{2} \theta}{\partial x^{2}}+\frac{\partial^{2} \theta}{\partial z^{2}}\right)=\left(\delta+\tau_{q} \frac{\partial}{\partial t}\right)\left[\frac{\partial \theta}{\partial t}+\frac{\gamma}{\eta K} \frac{\partial}{\partial t}\left(\frac{\partial u}{\partial x}+\frac{\partial w}{\partial z}\right)\right] \\
& (1-\nu) \frac{\partial^{2} u}{\partial x^{2}}+\frac{1-2 \nu}{2} \frac{\partial^{2} u}{\partial z^{2}}+\frac{1}{2} \frac{\partial^{2} w}{\partial x \partial z}-\alpha T_{0} \frac{\partial \theta}{\partial x}=\frac{\partial^{2} u}{\partial t^{2}} \\
& \frac{1}{2} \frac{\partial^{2} u}{\partial x \partial z}+\frac{1-2 \nu}{2} \frac{\partial^{2} w}{\partial x^{2}}+(1-\nu) \frac{\partial^{2} w}{\partial z^{2}}-\alpha T_{0} \frac{\partial \theta}{\partial z}=\frac{\partial^{2} w}{\partial t^{2}}
\end{aligned}
$$

In addition, the stress components will be

$$
\left\{\begin{array}{l}
\sigma_{x} \\
\sigma_{z}
\end{array}\right\}=\left[\begin{array}{cc}
1-\nu & \nu \\
\nu & 1-\nu
\end{array}\right]\left\{\begin{array}{l}
\frac{\partial u}{\partial x}-\alpha T_{0} \theta \\
\frac{\partial w}{\partial z}-\alpha T_{0} \theta
\end{array}\right\} \quad \sigma_{x z}=\frac{\partial w}{\partial x}+\frac{\partial u}{\partial z}
$$

\section{Solution of the problem}

To obtain the displacements, temperature and stresses of the beam, thermal and mechanical boundary conditions must be satisfied. Firstly, the following simply-supported conditions are imposed at the edges of the beam

$$
\sigma_{x}(x, z, t)=0 \quad w(x, z, t)=0 \quad \theta(x, z, t)=0 \quad \text { at } \quad x=0, L
$$


The closed form solution of the governing and constitutive equations may be obtained by adapting the supported-normal mode analysis as

$$
\{u, w, \theta\}(x, z, t)=\left\{u^{*}(z) \cos (\mu x), w^{*}(z) \sin (\mu x), \theta^{*}(z) \sin (\mu x)\right\} \mathrm{e}^{\omega t}
$$

where $\mu=\pi / L$. The displacement and temperature components given in Eq. (4.2) are satisfying the above boundary conditions on the edges of the beam. Then, Eqs. (3.6), after some elementary manipulations, become

$$
\begin{aligned}
& \left(\frac{d^{2}}{d z^{2}}-c_{1}\right) u^{*}+c_{2} \frac{d w^{*}}{d z}=c_{3} \theta^{*} \\
& \left(\frac{d^{2}}{d z^{2}}-c_{7}\right) \theta^{*}=c_{8} u^{*}+c_{9} \frac{d w^{*}}{d z}
\end{aligned} \quad\left(\frac{d^{2}}{d z^{2}}-c_{4}\right) w^{*}+c_{5} \frac{d u^{*}}{d z}=c_{6} \frac{d \theta^{*}}{d z}
$$

where the expressions $c_{k}$ are given by

$$
\begin{aligned}
& c_{1}=\frac{2\left[\omega^{2}+\mu^{2}(1-\nu)\right]}{1-2 \nu} \quad c_{2}=\frac{\mu}{1-2 \nu} \quad c_{3}=\frac{2 \mu \alpha T_{0}}{1-2 \nu} \\
& c_{4}=\frac{2 \omega^{2}+\mu^{2}(1-2 \nu)}{2(1-\nu)} \quad c_{5}=-\frac{\mu}{2(1-\nu)} \quad c_{6}=\frac{\alpha T_{0}}{1-\nu} \\
& c_{7}=\mu^{2}+\frac{\left(\delta+\tau_{q} \omega\right) \omega}{1+\tau_{\theta} \omega} \quad c_{8}=-\frac{\left(\delta+\tau_{q} \omega\right) \gamma \mu \omega}{\left(1+\tau_{\theta} \omega\right) \eta K} \quad c_{9}=\frac{\left(\delta+\tau_{q} \omega\right) \gamma \omega}{\left(1+\tau_{\theta} \omega\right) \eta K}
\end{aligned}
$$

In addition, the stresses are

$$
\left\{\sigma_{x}, \sigma_{z}\right\}=\left\{\sigma_{x}^{*}(z), \sigma_{z}^{*}(z)\right\} \mathrm{e}^{\omega t} \sin (\mu x) \quad \sigma_{x z}=\sigma_{x z}^{*}(z) \mathrm{e}^{\omega t} \cos (\mu x)
$$

They are given by

$$
\left\{\begin{array}{l}
\sigma_{x}^{*} \\
\sigma_{z}^{*}
\end{array}\right\}=\left[\begin{array}{cc}
1-\nu & \nu \\
\nu & 1-\nu
\end{array}\right]\left\{\begin{array}{c}
-\mu u^{*}-\alpha T_{0} \theta^{*} \\
\frac{d w^{*}}{d z}-\alpha T_{0} \theta^{*}
\end{array}\right\} \quad \sigma_{x z}^{*}=\mu w^{*}+\frac{d u^{*}}{d z}
$$

Eliminating $u^{*}(z)$ and $w^{*}(z)$ in Eqs. (4.3), one obtains

$$
\left(\frac{d^{6}}{d z^{6}}-A_{1} \frac{d^{4}}{d z^{4}}+A_{2} \frac{d^{2}}{d z^{2}}-A_{3}\right) \theta^{*}(z)=0
$$

where

$$
\begin{aligned}
& A_{1}=c_{1}+c_{4}+c_{7}+c_{2} c_{5}+c_{6} c_{9} \\
& A_{2}=c_{1}\left(c_{4}+c_{7}+c_{6} c_{9}\right)-c_{3}\left(c_{8}-c_{5} c_{9}\right)+c_{5}\left(c_{2} c_{7}+c_{3} c_{9}\right)+c_{4} c_{7} \\
& A_{3}=c_{4}\left(c_{1} c_{7}-c_{3} c_{8}\right)
\end{aligned}
$$

Now, Eq. (4.7) may be factorized as

$$
\left(\frac{d^{2}}{d z^{2}}-\lambda_{1}^{2}\right)\left(\frac{d^{2}}{d z^{2}}-\lambda_{2}^{2}\right)\left(\frac{d^{2}}{d z^{2}}-\lambda_{3}^{2}\right) \theta^{*}(z)=0
$$

where $\lambda_{j}^{2}(j=1,2,3)$ denote the roots of the characteristic equation

$$
\lambda^{6}-A_{1} \lambda^{4}+A_{2} \lambda^{2}-A_{3}=0
$$


They are given by

$$
\begin{aligned}
& \lambda_{1,2}=\mp \frac{\sqrt{\mathrm{i} \sqrt[3]{A_{0}}\left[(1+\mathrm{i} \sqrt{3}) \sqrt[3]{A_{0}}-4 A_{1}\right]+4(1-\mathrm{i} \sqrt{3})\left(A_{1}^{2}-3 A_{2}\right)}}{2 \sqrt{3} \sqrt[6]{A_{0}}} \\
& \lambda_{3,4}=\mp \frac{\sqrt{\sqrt[3]{A_{0}}\left[4 A_{1}-(1-\mathrm{i} \sqrt{3}) \sqrt[3]{A_{0}}\right]-4(1+\mathrm{i} \sqrt{3})\left(A_{1}^{2}-3 A_{2}\right)}}{2 \sqrt{3} \sqrt[6]{A_{0}}} \\
& \lambda_{5,6}=\mp \frac{\sqrt{\sqrt[3]{A_{0}}\left(\sqrt[3]{A_{0}}+2 A_{1}\right)+4\left(A_{1}^{2}-3 A_{2}\right)}}{\sqrt{6} \sqrt[6]{A_{0}}}
\end{aligned}
$$

where $\mathrm{i}=-1$ and

$$
A_{0}=8 A_{1}^{3}-36 A_{1} A_{2}+108 A_{3}+\sqrt{3 A_{1}^{2}\left(4 A_{1} A_{3}-A_{2}^{2}\right)-6 A_{2}\left(9 A_{1} A_{3}-2 A_{2}^{2}\right)+81 A_{3}^{2}}
$$

The solution $\theta^{*}(z)$ of Eq. (4.7) is given by

$$
\theta^{*}(z)=\sum_{j=1}^{3}\left(B_{1 j} \mathrm{e}^{\lambda_{j} z}+B_{2 j} \mathrm{e}^{-\lambda_{j} z}\right)
$$

where $B_{k j}(k=1,2)$ are arbitrary unknown complex constants connected with the boundary conditions. In a similar manner, one gets

$$
\left\{u^{*}(z), w^{*}(z)\right\}=\sum_{j=1}^{3}\left(\left\{\hat{B}_{1 j}, \check{B}_{1 j}\right\} \mathrm{e}^{\lambda_{j} z}+\left\{\hat{B}_{2 j}, \check{B}_{2 j}\right\} \mathrm{e}^{-\lambda_{j} z}\right)
$$

where $\hat{B}_{k j}$ and $\check{B}_{k j}$ are additional unknown constants. Substitution of Eqs. (4.13) and (4.14) into Eqs. $(4.3)_{1}$ and $(4.3)_{2}$ gives

$$
\left\{\hat{B}_{1 j}, \hat{B}_{2 j}\right\}=U_{\lambda_{j}}\left\{B_{1 j}, B_{2 j}\right\} \quad\left\{\check{B}_{1 j}, \check{B}_{2 j}\right\}=W_{\lambda_{j}}\left\{B_{1 j},-B_{2 j}\right\}
$$

in which

$$
U_{\lambda_{j}}=\frac{c_{3}\left(\lambda_{j}^{2}-c_{4}\right)-c_{2} c_{6} \lambda_{j}^{2}}{\left(\lambda_{j}^{2}-c_{1}\right)\left(\lambda_{j}^{2}-c_{4}\right)-c_{2} c_{5} \lambda_{j}^{2}} \quad W_{\lambda_{j}}=\frac{\lambda_{j}\left[c_{6}\left(\lambda_{j}^{2}-c_{1}\right)-c_{3} c_{5}\right]}{\left(\lambda_{j}^{2}-c_{1}\right)\left(\lambda_{j}^{2}-c_{4}\right)-c_{2} c_{5} \lambda_{j}^{2}}
$$

Finally, the stress amplitudes are

$$
\begin{aligned}
\sigma_{x}^{*} & =\sum_{j=1}^{3} \sigma_{\lambda_{j}}^{x}\left(B_{1 j} \mathrm{e}^{\lambda_{j} z}+B_{2 j} \mathrm{e}^{-\lambda_{j} z}\right) \\
\sigma_{x z}^{*} & =\sum_{j=1}^{3} \sigma_{\lambda j}^{x z}\left(B_{1 j} \mathrm{e}^{\lambda_{j} z}-B_{2 j} \mathrm{e}^{-\lambda_{j} z}\right)
\end{aligned}
$$

where

$$
\sigma_{\lambda j}^{x}=-\mu(1-\nu) U_{\lambda j}+\nu \lambda_{j} W_{\lambda j}-\alpha T_{0} \quad \sigma_{\lambda j}^{z}=-\mu \nu U_{\lambda j}+(1-\nu) \lambda_{j} W_{\lambda j}-\alpha T_{0}
$$

Now, the upper surface of the beam is considered to be thermally insulated and subjected to a mechanical load while the bottom surface is traction free and subjected to a heating source (Zenkour and Abouelregal, 2016). So, the beam is subjected to the following boundary conditions

$$
\begin{array}{lr}
\theta\left(x,-\frac{h}{2}, t\right)=\left.\theta_{0} \sin (\mu x) \mathrm{e}^{\omega t} \quad \frac{\partial \theta}{\partial z}\right|_{z=+\frac{h}{2}}=0 \\
\sigma_{z}\left(x,-\frac{h}{2}, t\right)=\sigma_{x z}\left(x, \pm \frac{h}{2}, t\right)=0 & \sigma_{z}\left(x, \frac{h}{2}, t\right)=-\sigma_{0} \sin (\mu x) \mathrm{e}^{\omega t}
\end{array}
$$


Substituting Eqs. (4.13), (4.14) and (4.17) into the above boundary conditions, one obtains six linear equations in the following matrix form

$$
\beta \mathbf{B}=\mathbf{G}
$$

where $\mathbf{G}=\left\{\theta_{0}, 0,0,-\sigma_{0}, 0,0\right\}^{\mathrm{T}}$ and $\mathbf{B}=\left\{B_{11}, B_{12}, B_{13}, B_{21}, B_{22}, B_{23}\right\}^{\mathrm{T}}$ is the vector of constants. The elements $\beta_{k j}^{l}(l=1,2, \ldots, 6)$ of the marix $\boldsymbol{\beta}$ are given by

$$
\begin{array}{lll}
\beta_{1 j}^{1}=\mathrm{e}^{-\lambda_{j} \frac{h}{2}} \quad \beta_{2 j}^{1}=\mathrm{e}^{\lambda_{j} \frac{h}{2}} \quad \beta_{1 j}^{2}=\lambda_{j} \beta_{2 j}^{1} \quad & \beta_{2 j}^{2}=-\lambda_{j} \beta_{1 j}^{1} \\
\beta_{1 j}^{3}=\beta_{2 j}^{4}=\sigma_{\lambda j}^{z} \mathrm{e}^{-\lambda_{j} \frac{h}{2}} & \beta_{2 j}^{3}=\beta_{1 j}^{4}=\sigma_{\lambda j}^{z} \mathrm{e}^{\lambda_{j} \frac{h}{2}} \\
\beta_{1 j}^{5}=-\beta_{2 j}^{6}=\sigma_{\lambda j}^{x z} \mathrm{e}^{-\lambda_{j} \frac{h}{2}} & \beta_{2 j}^{5}=-\beta_{1 j}^{6}=-\sigma_{\lambda j}^{x z} \mathrm{e}^{\lambda_{j} \frac{h}{2}}
\end{array}
$$

Solving the system of above equations to get values of the constants $B_{k j}$. So, this completes the solution of the problem. Hence, one can easily obtains expressions for the dimensionless quantities of temperature $\theta$, displacements $u$ and $w$, and stresses $\sigma_{1}=\sigma_{x}, \sigma_{3}=\sigma_{z}$ and $\sigma_{5}=\sigma_{x z}$ in the present beam.

\section{Numerical results}

The thermoelastic coupling effect is presented here to get the temperature, displacements and stresses. The material parameters used here are due to physical data of copper at $T_{0}=293 \mathrm{~K}$ : $\lambda^{*}=7.76 \cdot 10^{10} \mathrm{~N} / \mathrm{m}^{2}, \mu^{*}=3.86 \cdot 10^{10} \mathrm{~N} / \mathrm{m}^{2}, \rho=8954 \mathrm{~kg} / \mathrm{m}^{3}, C^{e}=383.1 \mathrm{~J} /(\mathrm{kg} \mathrm{K})$, $\left.K=386 \mathrm{~N} /(\mathrm{s} \mathrm{K}), \alpha=1.78 \cdot 10^{-5} \mathrm{~K}^{-1}\right)$.

It is to be noted that Young's modulus $E$ and Poisson's ratio $\nu$ are given in terms of Lamé's constants $\lambda^{*}$ and $\mu^{*}$ by

$$
E=\frac{\mu^{*}\left(3 \lambda^{*}+2 \mu^{*}\right)}{\lambda^{*}+\mu^{*}} \quad \nu=\frac{\lambda^{*}}{2\left(\lambda^{*}+\mu^{*}\right)}
$$

The length-to-thickness ratio of the beam is fixed at $L / h=5$ and the angular frequency $\omega=\omega_{0}+\mathrm{i} \zeta$. All plots are prepared by using the real values of the dimensionless variables defined in Eq. (3.5) for a wide range of the beam length and thickness. The computations are carried out for different values of time and delay time parameters $\tau_{\theta}$ and $\tau_{q}$. Once again, the directions of the beam are given in terms of the length and thickness of the beam, that is $\bar{x}=x / L$ and $\bar{z}=z / h$ (the prime is dropped in the figures for convenience). Figures 2-7 compare the results obtained for temperature, displacements and stresses against the $x$ and $z$ directions when $t=0.3$. The variation of the field quantities versus the time parameter are also presented in Figs. 8-10. The graphs represent curves predicted by the CTE, L-S and G-N models of thermoelasticity obtained as special cases of the present general DPL model. The results of the CTE model $\left(\tau_{\theta}=\tau_{q}=0, \delta=1\right)$, the L-S model $\left(\tau_{\theta}=0, \tau_{q}=0.05, \delta=1\right)$, the G-N model $\left(\tau_{\theta}=0, \tau_{q}=0.05, \delta=0\right)$, and the DPL model $\left(\tau_{\theta}=0.02<\tau_{q}=0.05, \delta=1\right)$ are all presented. Also, other parameters are fixed at $\theta_{0}=0.5, \omega_{0}=2, \zeta=-1$, and two values are considered for the intensity of the applied load, namely $\sigma_{0}=0.5$ and $\sigma_{0}=1$.

Figure 2a shows the variation of the dimensionless temperature $\theta$ along the axial direction at the upper surface $z=0.5$ of the beam. The behavior of all models may be the same with different amplitudes. The absolute maximum temperature occurs at the center of the beam. For the two load cases, the temperature of the L-S model is positive for $\sigma_{0}=0.5$ while it changes to negative for $\sigma_{0}=1$. The DPL model gives the largest temperatures while L-S model gives the smallest ones. 
Figure $2 \mathrm{~b}$ shows the variation of the dimensionless axial displacement $u$ along the axial direction at the mid-plane $z=0$ of the beam. The axial displacement, as expected, vanishes at the center of the beam for all models. The CTE model gives the smallest axial displacements at the first edge of the beam and the smallest ones at the second edge. However, the DPL model gives the largest axial displacements at the first edge of the beam and the smallest ones at the second edge. The behavior of the L-S model may be unchanged for the two cases of lateral loads.

(a)

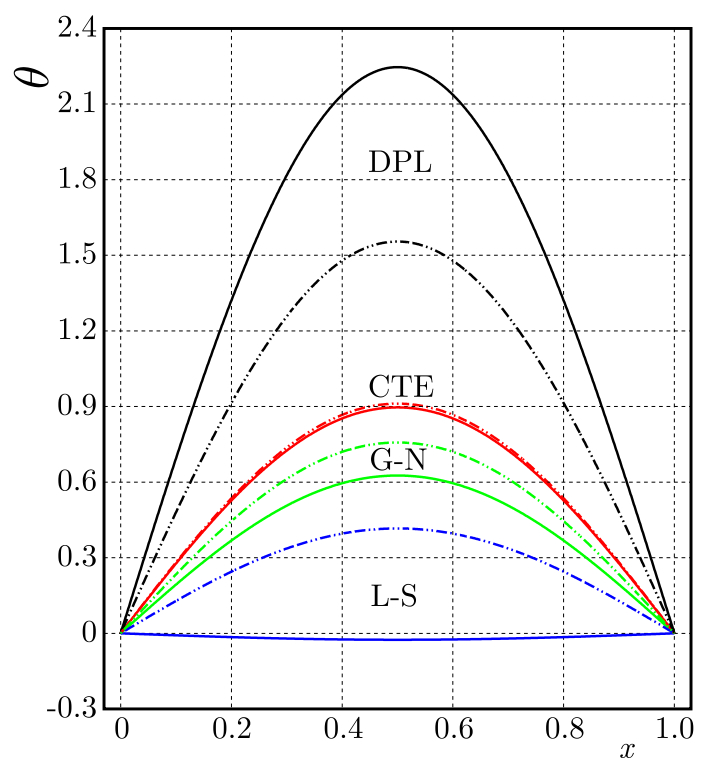

(b)

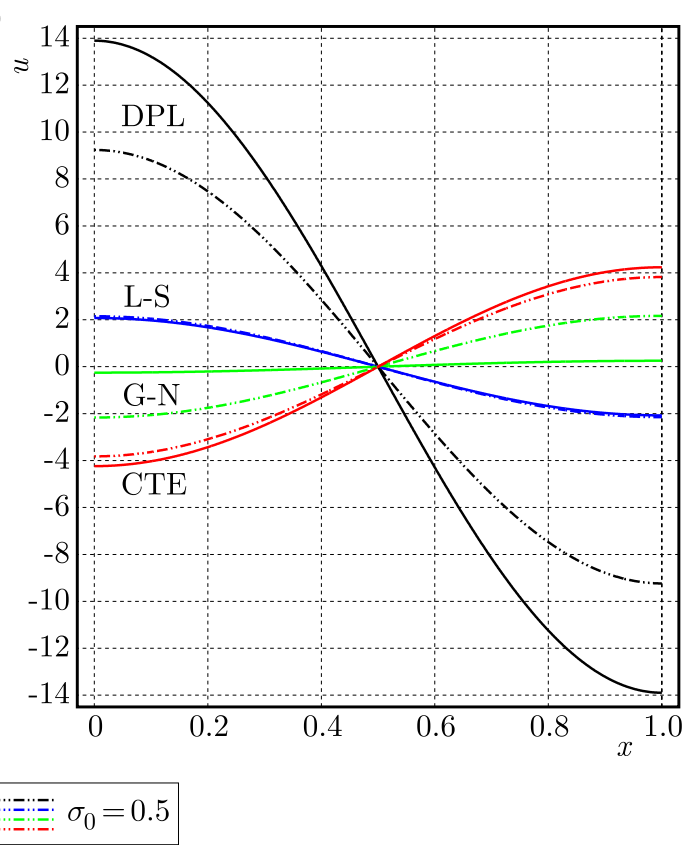

Fig. 2. Distribution of temperature $\theta$ (a) and of axial displacement $u$ (b) in the axial direction for two load parameters

Figure 3a shows the variation of the transverse displacement $w$ along the axial direction at the middle surface $z=0$. Each model gives different behavior of $w$ along the axial direction of the beam. The maximum (minimum) deflection occurs at the center of the beam for the L-S model (CTE model) in the two cases $\sigma_{0}=1$ and $\sigma_{0}=0.5$.

Figure $3 \mathrm{~b}$ shows the variation of the dimensionless axial stress $\sigma_{1}$ along the axial direction at the upper surface $z=0.5$ of the beam. The absolute maximum axial stress $\sigma_{1}$ occurs at the center of the beam $(x=0.5)$. For the first loaded beam $\left(\sigma_{0}=0.5\right)$, the axial stress $\sigma_{1}$ of the G-N model only still positive while other models give negative axial stresses along the axial direction for the two loaded beams.

Figure 4 a shows the variation of the dimensionless normal stress $\sigma_{3}$ along the axial direction at the upper surface $z=0.5$ of the beam. All of the transverse normal stresses are compressive due to different models. The DPL model gives the smallest normal stress when $\sigma_{0}=1$ and the largest ones when $\sigma_{0}=0.5$.

Figure $4 \mathrm{~b}$ shows the variation of the dimensionless transverse shear stress $\sigma_{5}$ along the axial direction at the mid-plane $z=0$ of the beam. The shear stresses vanish at the center of the axial direction according to all models. The shear stresses for $\sigma_{0}=0.5$ are much smaller than those for $\sigma_{0}=1$. The DPL and L-S models give tensile shear stresses at the first edge and compressive shear stresses at the second edge of the beam for both $\sigma_{0}=0.5$ and $\sigma_{0}=1$. Also, the CTE model gives compressive shear stresses at the first edge and tensile shear stresses at the second edge of the beam for both $\sigma_{0}=0.5$ and $\sigma_{0}=1$. However, the G-N model gives compressive shear stress at the first edge and tensile shear stresses at the second edge of the beam for $\sigma_{0}=1$ and vice versa for $\sigma_{0}=0.5$. 
(a)

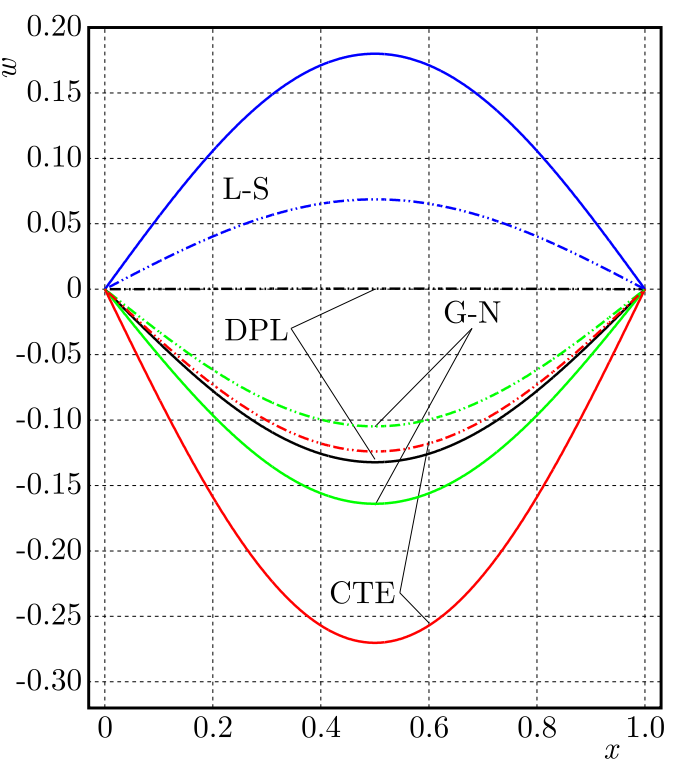

(b)

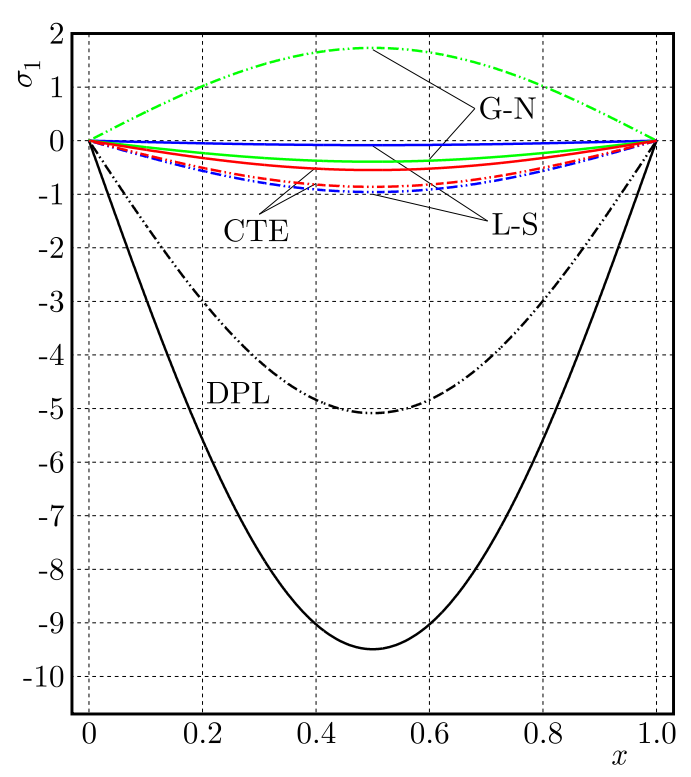

$\bar{\equiv} \sigma_{0}=1.0 \quad: \because::: \equiv: \sigma_{0}=0.5$

Fig. 3. Distribution of transverse deflection $w$ (a) and of axial stress $\sigma_{1}$ (b) in the axial direction for two load parameters

(a)

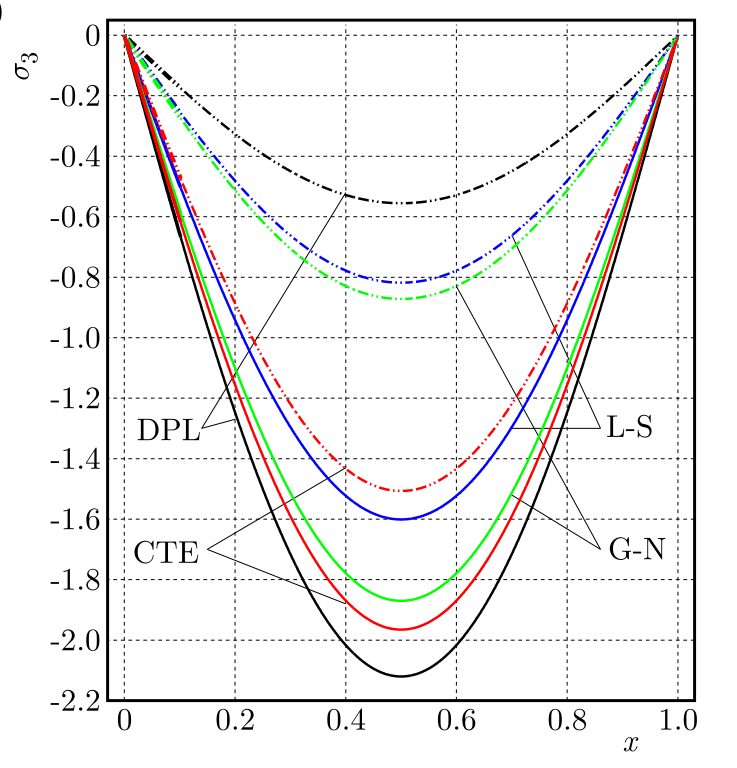

(b)

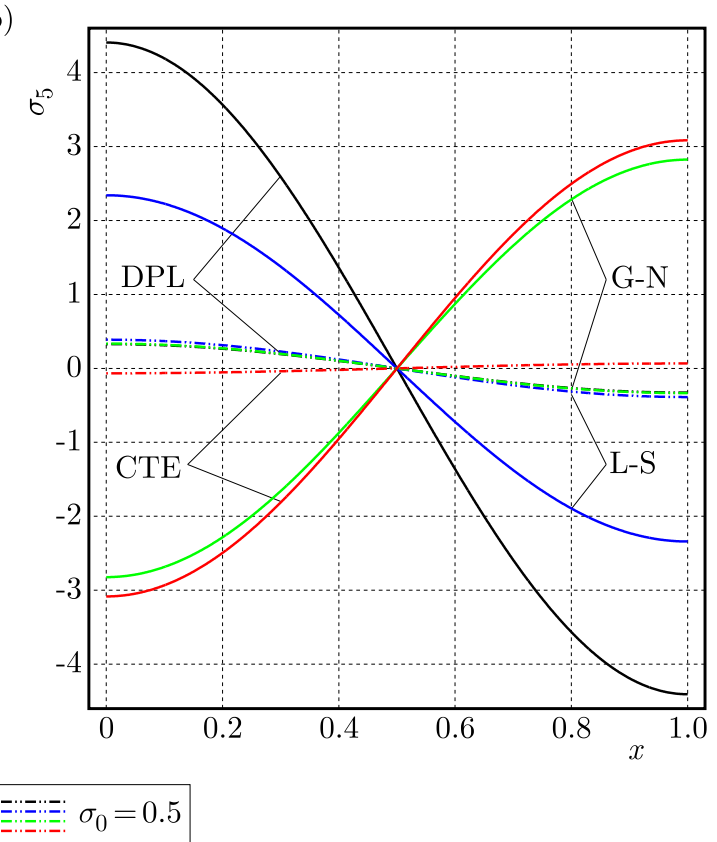

Fig. 4. Distribution of transverse normal stress $\sigma_{3}$ (a) and of transverse shear stress $\sigma_{5}$ in the axial direction for two load parameters

Figure 5a shows the through-the-thickness variation of the dimensionless temperature $\theta$ at the center $x=0.5$ of the beam. The maximum temperature occurs at the upper face of the beam according to all models due to thermal conditions. The temperature for the CTE model may change through the beam thickness with a very small magnitude comparing to other models. All models, as expected, have the same temperature at the bottom surface of the beam. However, the DPL and L-S models give, respectively, the largest and smallest temperature at the upper surface of the beam. 
Figure 5b shows the through-the-thickness variation of the axial displacement $u$ at the first edge $x=0$ of the beam under various loads. All models give different behavior of the axial displacements. The axial displacements for the DPL model are positive, and for the CTE and G-N models are negative.

(a)

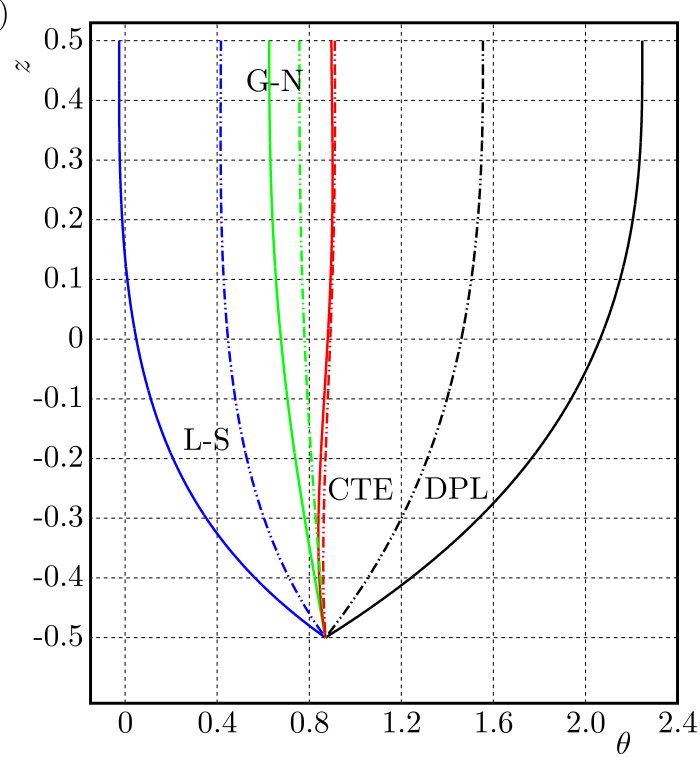

(b)

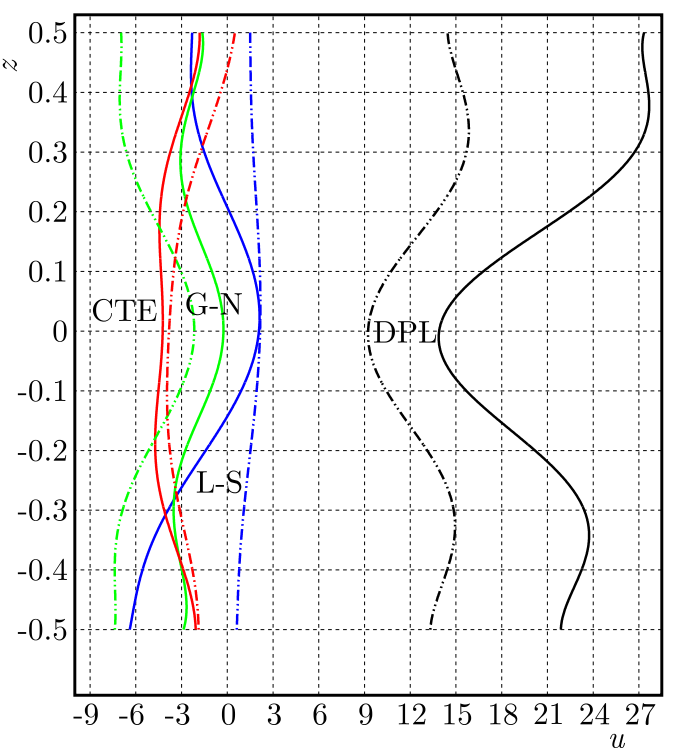

$\bar{\equiv} \sigma_{0}=1.0 \quad: \vdots::::::: \sigma_{0}=0.5$

Fig. 5. Distribution of temperature $\theta$ (a) and of axial displacement $u$ (b) in the thickness direction for two load parameters

(a)

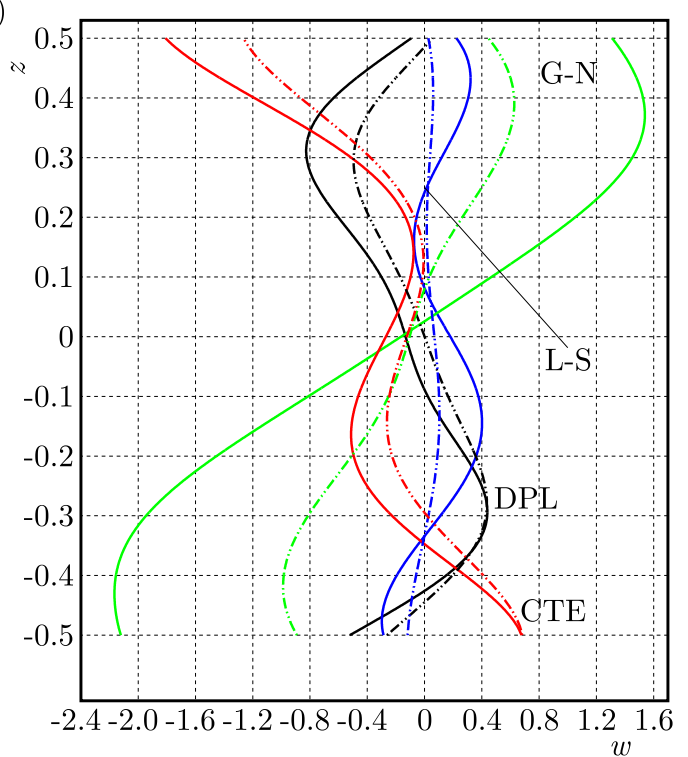

(b)

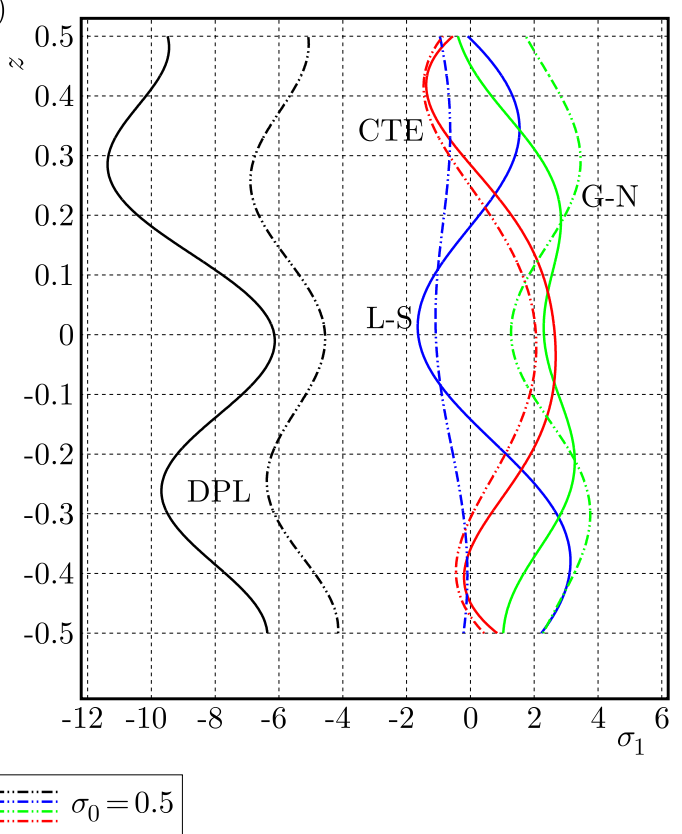

Fig. 6. Distribution of transverse deflection $w$ (a) and of axial stress $\sigma_{1}$ (b) in the thickness direction for two load parameters

Figure 6a shows the through-the-thickness variation of the dimensionless transverse displacement $w$ at the center $x=0.5$ of the beam. The deflections due to the DPL and L-S models may be closed to each other. All models may exhibit different behavior through-the-thickness of the beam. 
Figure $6 \mathrm{~b}$ shows the through-the-thickness variation of the dimensionless axial stress $\sigma_{1}$ at the center of the beam $x=0.5$. The DPL model gives axial stresses more different than those of other models. The axial stresses due to the L-S, G-N and CTE are close to each other. The axial stresses for the DPL when $\sigma_{0}=0.5$ are greater than those of the DPL model when $\sigma_{0}=1$. This is not the same for other models.

Figure 7a shows the through-the-thickness variation of the dimensionless normal stress $\sigma_{3}$ at the center of the beam $x=0.5$. All models are very sensitive to the variation of the used load.

Figure $7 \mathrm{~b}$ shows the through-the-thickness variation of the dimensionless transverse shear stress $\sigma_{5}$ at the first edge $x=0$ of the beam. All models are very sensitive to the variation of the used load, especially in the DPL model. The DPL model gives the smallest compressive stress near the mid-plane of the beam at $z=-0.16$ and tensile axial stress at $z=-0.16$.

(a)

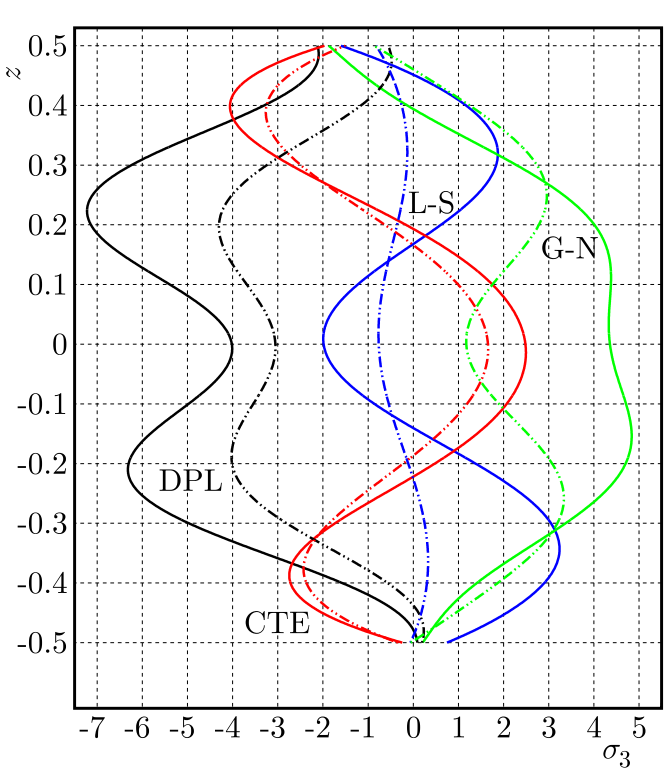

(b)

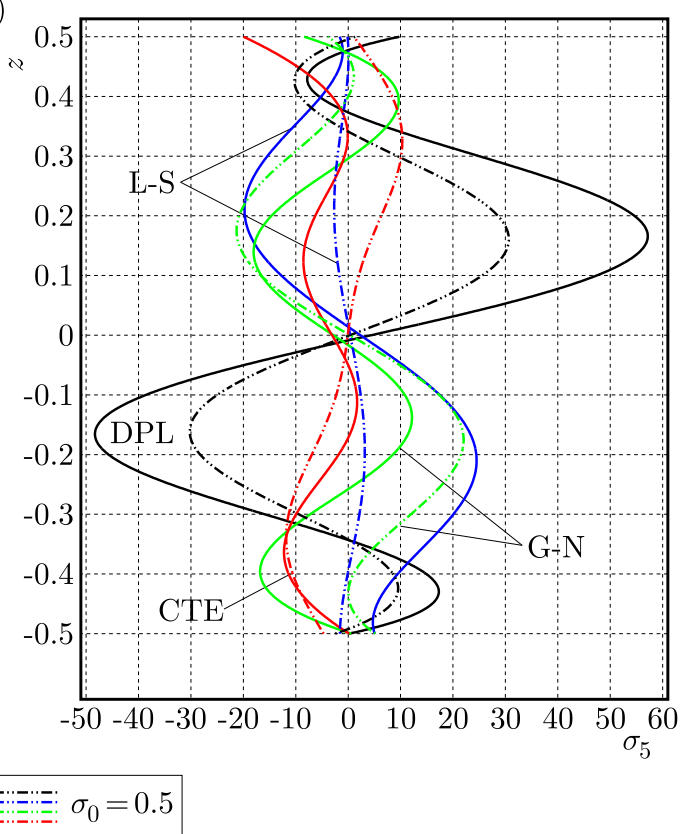

Fig. 7. Distribution of transverse normal stress $\sigma_{3}$ (a) and of transverse shear stress $\sigma_{5}$ in the thickness direction for two load parameters

Figure $8 \mathrm{a}$ shows the variation of the dimensionless temperature $\theta$ versus the time parameter at the center $(x=0.5$ and $z=0)$ of the beam. The behavior of all models may be different. The sign of temperature for all models (except the G-N model) may be changed from positive to negative. At $t=1.5$, the CTE model gives the smallest temperature and the G-N model gives the largest temperature for $\sigma_{0}=1$. Also, temperatures for the G-N (L-S) model are directly increasing (decreasing) as $t$ increases. Otherwise, the temperatures are no longer increasing and have their maximum at different values of the time parameter.

Figure $8 \mathrm{~b}$ shows the variation of the dimensionless axial displacement $u$ versus the time parameter at the first edge $x=0$ of the mid-plane $z=0$ of the beam under two different loads. The axial displacement for the DPL (G-N) model are directly increasing (decreasing) as $t$ increases for the two cases $\sigma_{0}=0.5$ and $\sigma_{0}=1$. The axial displacement for the CTE model are directly increasing as $t$ increases for $\sigma_{0}=0.5$ and decreasing as $t$ increases for $\sigma_{0}=1$.

Figure 9a shows the variation of the transverse displacement $w$ versus the time parameter at the center $(x=0.5$ and $z=0)$ of the beam. The deflections for the L-S model are directly increasing as $t$ increases for the two cases $\sigma_{0}=0.5$ and $\sigma_{0}=1$. However, the deflections for the other models are directly decreasing as $t$ increases for the two cases $\sigma_{0}=0.5$ and $\sigma_{0}=1$. 
(a)

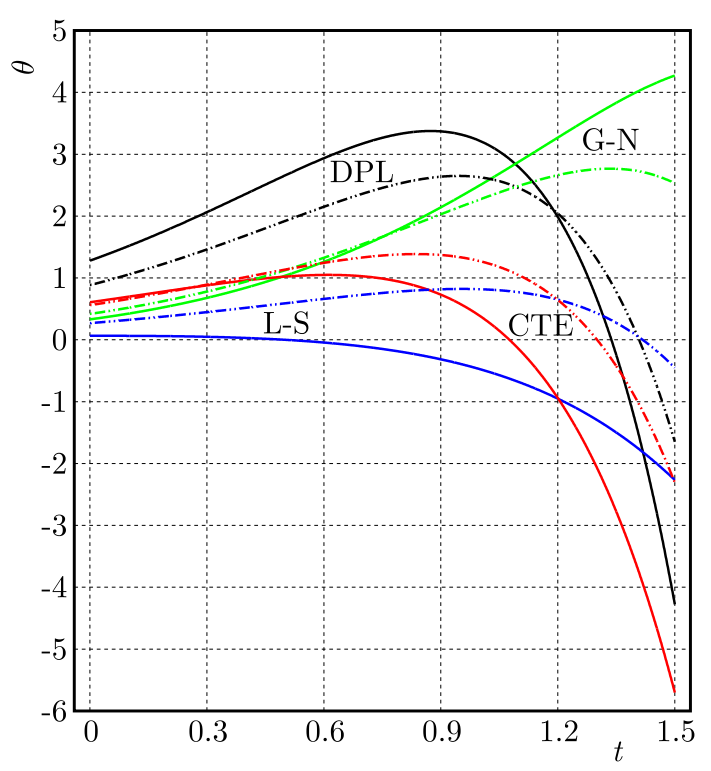

(b)

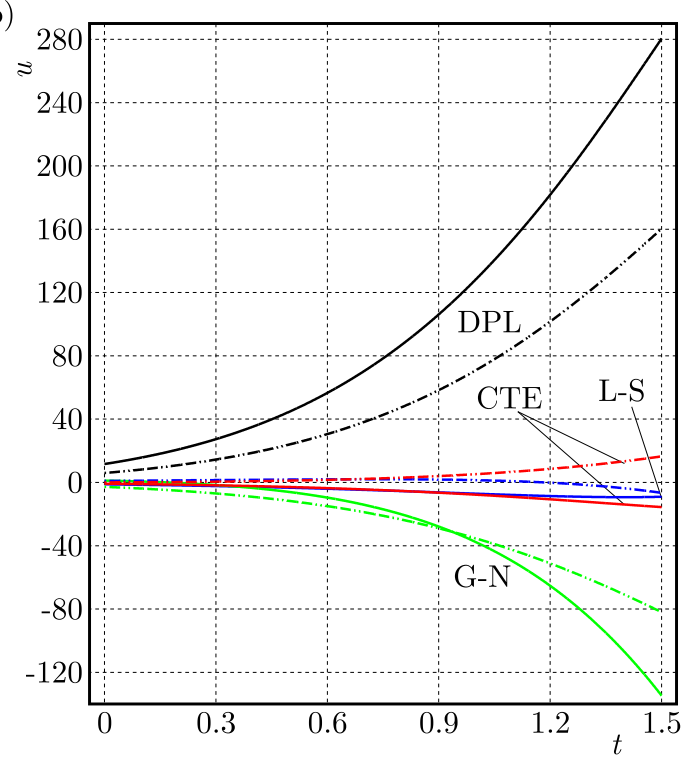

$\bar{\equiv} \sigma_{0}=1.0 \quad \Xi \equiv: \sigma_{0}=0.5$

Fig. 8. Variation of temperature $\theta$ (a) and of axial displacement $u$ (b) versus the time parameter for two load parameters

Figure $9 \mathrm{~b}$ shows the variation of the dimensionless axial stress $\sigma_{1}$ versus the time parameter at the center $x=0.5$ of the upper surface $z=0.5$ of the beam. The axial stress for the G-N (DPL) model are directly increasing (decreasing) as $t$ increases for the two cases $\sigma_{0}=0.5$ and $\sigma_{0}=1$. The axial stress for the CTE model are directly decreasing as $t$ increases for $\sigma_{0}=0.5$.

(a)

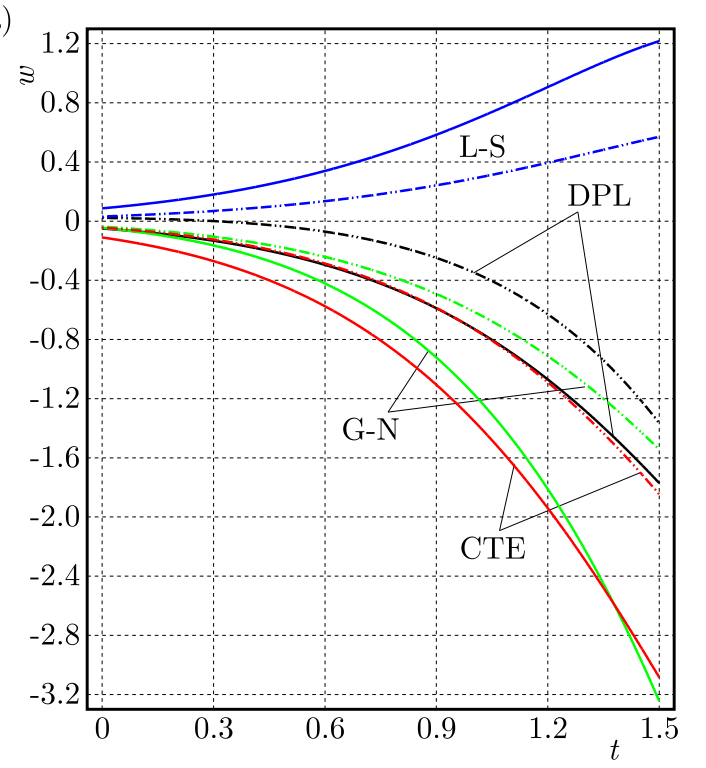

(b)

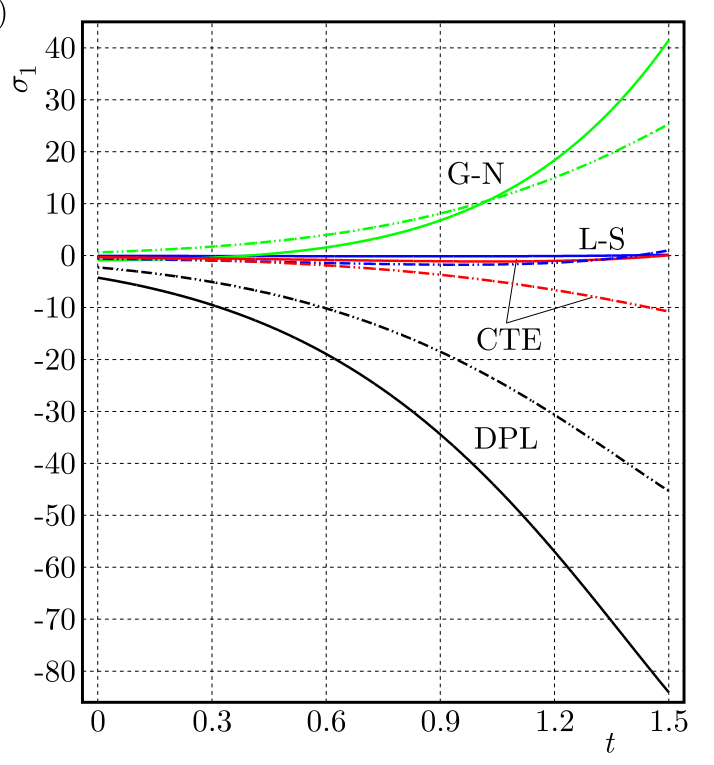

$\bar{\equiv} \sigma_{0}=1.0 \quad \Xi \vdots: \cdots: \sigma_{0}=0.5$

Fig. 9. Variation of transverse deflection $w$ (a) and of axial stress $\sigma_{1}$ (b) versus the time parameter for two load parameters

Figure 10a shows the variation of the dimensionless normal stress $\sigma_{3}$ versus the time parameter at the center $x=0.5$ of the upper surface $z=0.5$ of the beam. Most transverse normal stresses are no longer decreasing, and finally increase as $t$ increases. For $\sigma_{0}=0.5, \sigma_{3}$ in the DPL 
model is directly increasing with an increase in the time parameter while $\sigma_{3}$ for the CTE model is directly decreasing.

Figure 10b shows the variation of the dimensionless transverse shear stress $\sigma_{5}$ versus the time parameter at the first edge of the mid-plane $(x=0, z=0)$ of the beam. The shear stresses for the L-S (G-N) model are increasing (decreasing) as $t$ increases for $\sigma_{0}=1$ and $\sigma_{0}=0.5$. For $\sigma_{0}=0.5, \sigma_{5}$ for the DPL (CTE) model is decreasing (increasing) as $t$ increases. For $\sigma_{0}=1$, $\sigma_{5}$ for the DPL model is no longer increasing and its maximum is at $t=1.1$, and then it is decreasing again while $\sigma_{5}$ for the CTE model is decreasing as $t$ increases.

(a)

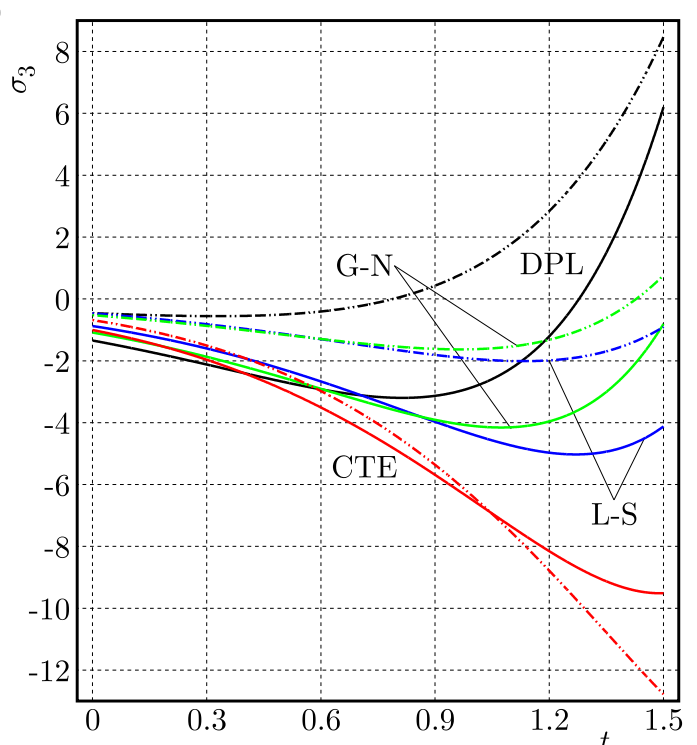

(b)

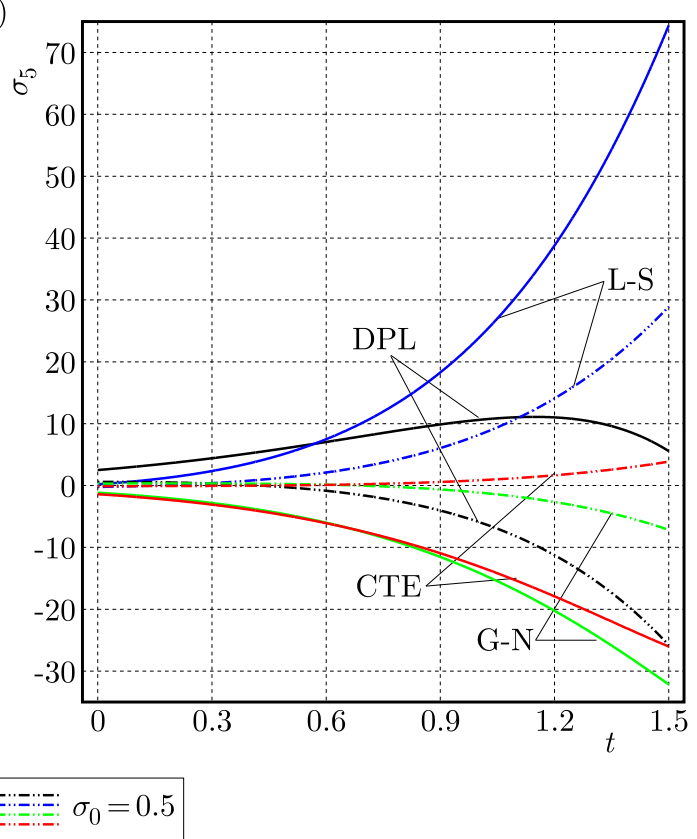

Fig. 10. Variation of transverse normal stress $\sigma_{3}$ (a) and of transverse shear stress $\sigma_{5}$ (b) versus the time parameter for two load parameters

For the sake of completeness and comparison, some plots for the filed quantities are displayed through-the-thickness of the beam using the DPL model only. Here, the upper surface of the beam is considered to be thermally insulated and subjected to a mechanical load while the bottom surface is subjected to both mechanical load and heating source. So, the beam is subjected to the following boundary conditions

$$
\begin{array}{ll}
\theta\left(x,-\frac{h}{2}, t\right)=\theta_{0} \sin (\mu x) \mathrm{e}^{\omega t} & \left.\frac{\partial \theta}{\partial z}\right|_{z=+\frac{h}{2}}=0 \\
\sigma_{5}\left(x, \pm \frac{h}{2}, t\right)=0 & \sigma_{3}\left(x,-\frac{h}{2}, t\right) \quad\left\{\sigma_{3}\left(x, \frac{h}{2}, t\right)\right\}=\left\{-\bar{\sigma}_{0}, \bar{\sigma}_{0}\right\} \sin (\mu x) \mathrm{e}^{\omega t}
\end{array}
$$

In this case, the initial temperature and stresses are fixed as $\theta_{0}=1, \bar{\sigma}_{0}=0.25$ and $\bar{\sigma}_{0}=0.75$. Figure 11a shows the through-the-thickness variation of the dimensionless temperature $\theta$ at the center $x=0.5$ of the beam according to different time parameters. The temperature increases with an increase in $z$ and $t$. Figure 11b shows the through-the-thickness variation of the axial displacement $u$ at the first edge $x=0$ of the beam according to different time parameters. The axial displacement is very sensitive to variation of the time parameter. The magnitude of the axial displacement wave is increasing as $t$ increases.

Figure 12a shows the through-the-thickness variation of the dimensionless transverse displacement $w$ at the center $x=0.5$ of the beam according to different time parameters. Also, the 

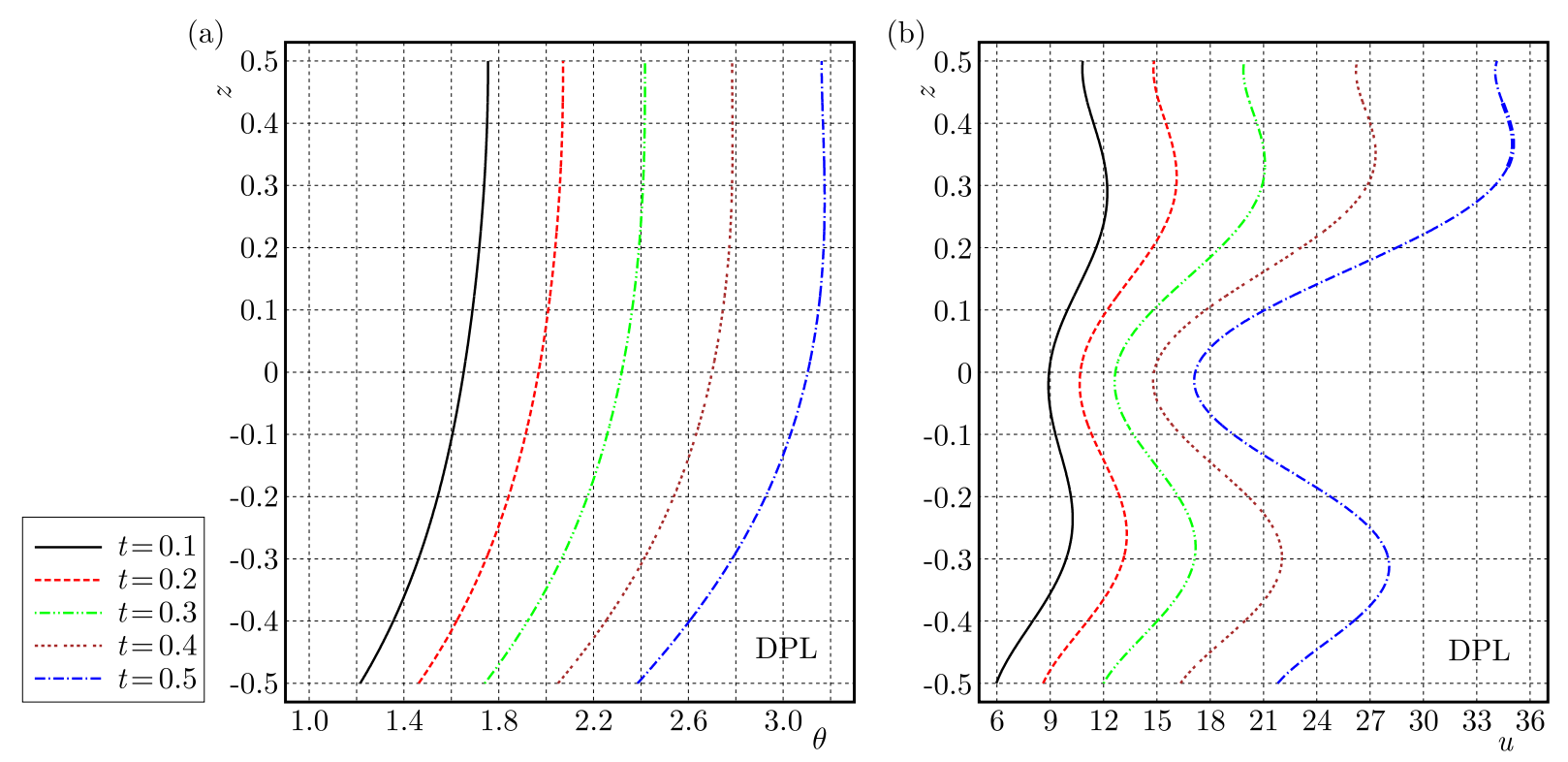

Fig. 11. Distribution of temperature $\theta$ (a) and of axial displacement $u$ (b) through-the-thickness of the beam at different time parameters
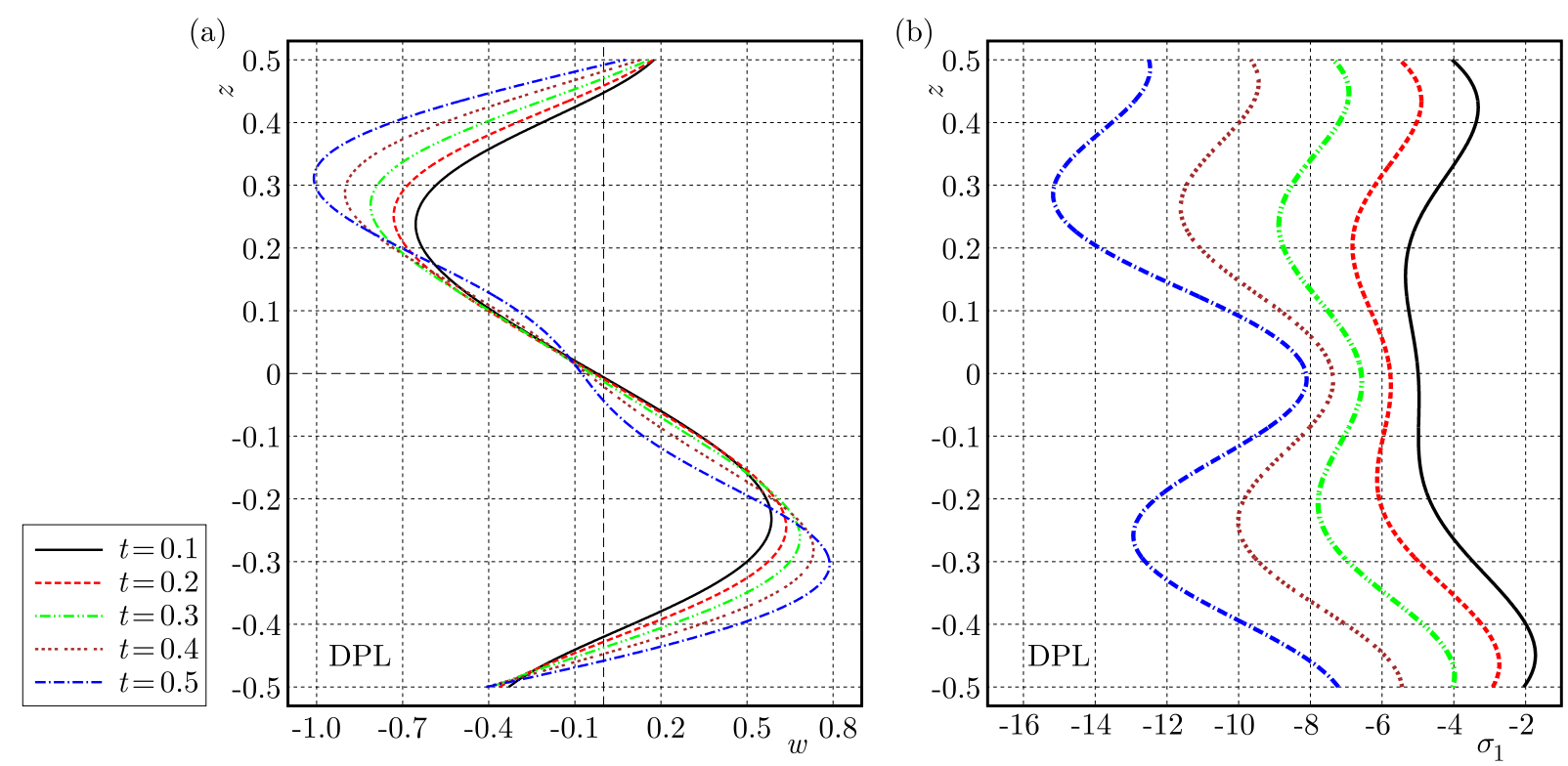

Fig. 12. Distribution of transverse deflection $w$ (a) and of axial stress $\sigma_{1}$ (b) through-the-thickness of the beam at different time parameters

deflection is very sensitive to variation of the time parameter. The magnitude of the deflection wave is increasing as $t$ increases. Figures $12 \mathrm{~b}$ and $13 \mathrm{a}$ show the through-the-thickness variation of the dimensionless axial stress $\sigma_{1}$ and the transverse normal stress $\sigma_{3}$ at the center $x=0.5$ of the beam according to different time parameters. The stresses themselves are decreasing as $t$ increases while the magnitudes of their waves are increasing. Finally, Figure 13b shows the through-the-thickness variation of the dimensionless transverse shear stress $\sigma_{5}$ at the first edge $x=0$ of the beam according to different time parameters. The shear stress is very sensitive to variation of the time parameter. The magnitude of the transverse shear stress wave is increasing as $t$ increases. 

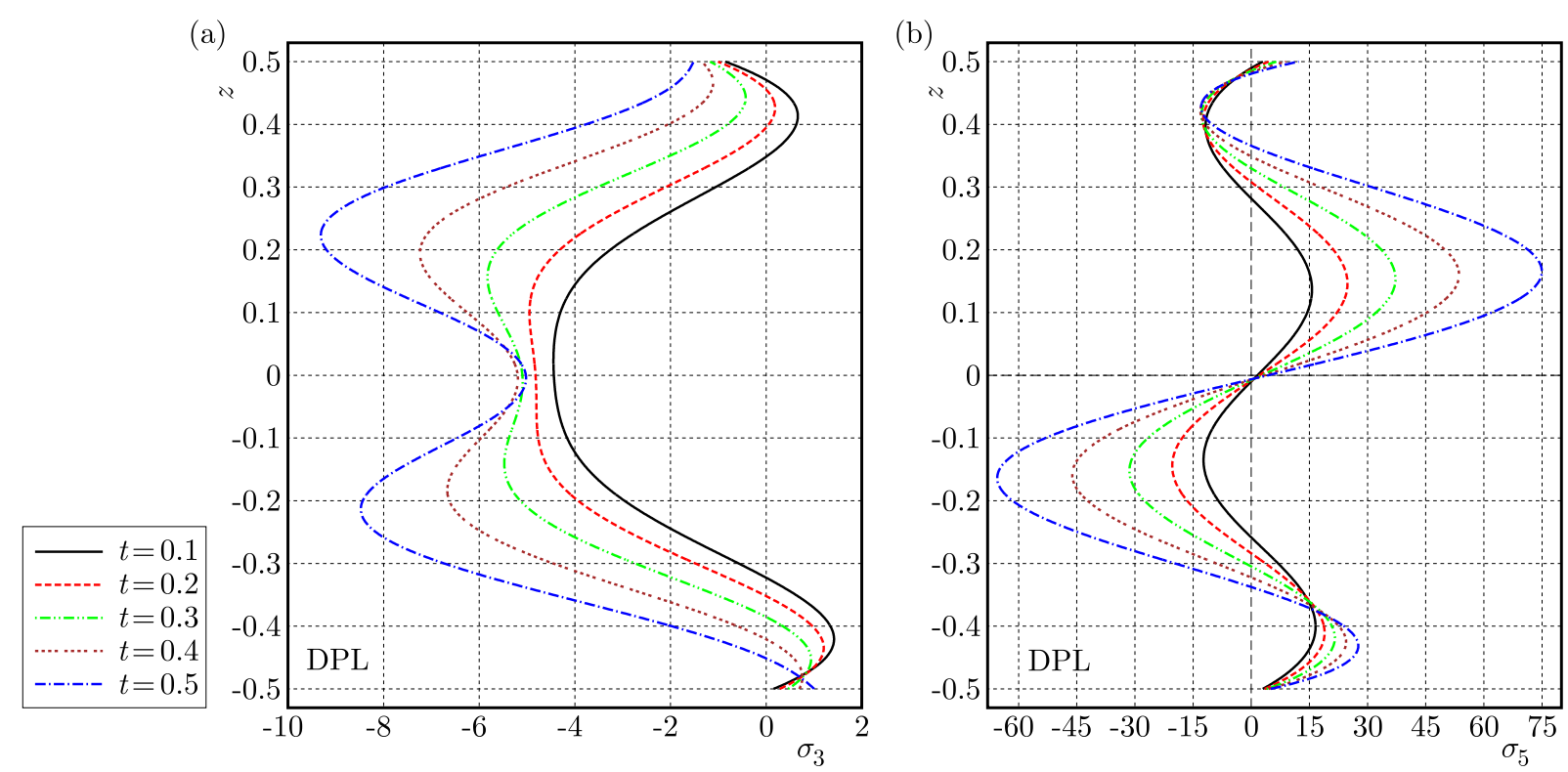

Fig. 13. Distribution of $\sigma_{3}$ (a) and $\sigma_{5}$ (b) through-the-thickness of the beam at different time parameters

\section{Conclusions}

The exact presentations of temperature, displacements and stresses in the axial and thickness directions of a generalized thermoelastic beam are considered in this article. The model of generalized thermoelasticity with dual-phase-laggings is constructed and other known thermoelastic models may be considered as special cases. The exact $2 \mathrm{D}$ general solution is applied to the present beam subjected to various heating sources or thermomechanical loads. The comparisons are shown along the axial and thickness directions of the beam. The field quantities are very sensitive to the applied thermal and mechanical loads and variation of the time parameter. The method used here may be applicable to a wide range of problems in thermodynamics and thermoelasticity. The numerical results presented here may be considered as more general in the sense that they include exact analysis of different field quantities. It is concluded from the graphical results presented here that the effect of dual-phase-lag parameters plays a significant role on all the physical quantities. Some models may fail to treat the thermoelastic response of many structures.

\section{References}

1. Abbas I.A., Zenkour A.M., 2014, Dual-phase-lag model on thermoelastic interactions in a semi-infinite medium subjected to a ramp-type heating, Journal of Computational and Theoretical Nanoscience, 11, 642-645

2. Abouelregal A.E., Zenkour A.M., 2014, Effect of phase lags on thermoelastic functionally graded microbeams subjected to ramp-type heating, Iranian Journal of Science and Technology, Transactions of Mechanical Engineering 38, 321-335

3. Allam M.N., Elsibai K.A. Abouelregal A.E., 2009, Electromagneto-thermoelastic problem in a thick plate using Green and Naghdi theory, International Journal of Engineering Science, 47, 680-690

4. Biot M.A., 1956, Thermoelasticity and irreversible thermodynamics, Journal of Applied Physics, 27, 240-253

5. Chandrasekharaiah D.S., 1998, Hyperbolic thermoelasticity: a review of recent literature, $A p$ plied Mechanics Reviews, 51, 705-729 
6. Green A.E., Lindsay K.A., 1972, Thermoelasticity, Journal of Elasticity, 2, 1-7

7. Green A.E., Naghdi P.M., 1991, A re-examination of the basic postulates of thermomechanics, Proceedings of the Royal Society, 432, 171-194

8. Green A.E., Naghdi P.M., 1992, On undamped heat waves in an elastic solid, Journal of Thermal Stresses, 15, 253-264

9. Green A.E., NAGhdi P.M., 1993, Thermoelasticity without energy dissipation, Journal of Elasticity, 31, 189-209

10. Guo F.L., Wang G.Q., Rogerson G.A., 2012, Analysis of thermoelastic damping in microand nano-mechanical resonators based on dual-phase-lagging generalized thermoelasticity theory, International Journal of Engineering Science, 60, 59-65

11. Kobzar' V.N., Fil'shtinskim L.A., 2008, The plane dynamic problem of coupled thermoelasticity, Journal of Applied Mathematics and Mechanics, 72, 611-618

12. Lord H.W., Shulman Y., 1967, A generalized dynamical theory of thermoelasticity, Journal of the Mechanics and Physics of Solids, 15, 299-309

13. MukHopadhyAY S., 2004, Thermoelastic interactions without energy dissipation in an unbounded body with a spherical cavity subjected to harmonically varying temperature, Mechanics Research Communications, 31, 81-89

14. Mukhopadhyay S., Kumar R., 2008, A study of generalized thermoelastic interactions in an unbounded medium with a spherical cavity, Applied Mathematics and Computation, 56, 2329-2339

15. Prasad R., Kumar R., Mukhopadhyay S., 2010, Propagation of harmonic plane waves under thermoelasticity with dual-phase-lags, International Journal of Engineering Science, 48, 2028-2043

16. Ram P., Sharma N., Kumar R., 2008, Thermomechanical response of generalized thermoelastic diffusion with one relaxation time due to time harmonic sources, International Journal of Thermal Sciences, 47, 315-323

17. Tzou D.Y., 1995a, A unified approach for heat conduction from macro- to micro-scales, Journal of Heat Transfer, 117, 8-16

18. Tzou D.Y., 1995b, Experimental support for the Lagging behavior in heat propagation, Journal of Thermophysics and Heat Transfer, 9, 686-693

19. Tzou D.Y., 1996, Macro-to-Microscale Heat Transfer: The Lagging Behavior, Washington, DC, Taylor \& Francis

20. Zenkour A.M., 2015, Three-dimensional thermal shock plate problem within the framework of different thermoelasticity theories, Composite Structures, 132, 1029-1042

21. Zenkour A.M., Abouelregal A.E., 2014, Nonlocal thermoelastic vibrations for variable thermal conductivity nanobeams due to harmonically varying heat, Journal of Vibroengineering, 16, $3665-3678$

22. Zenkour A.M., Abouelregal A.E., 2015, The nonlocal dual phase lag model of thermoelastic nanobeam subjected to a sinusoidal pulse heating, Journal for Computational Methods in Engineering Science and Mechanics, 16, 53-73

23. Zenkour A.M., Abouelregal A.E., 2016, Non-simple magnetothermoelastic solid cylinder with variable thermal conductivity due to harmonically varying heat, Earthquakes and Structures, 10, 681-697

24. Zenkour A.M., Mashat D.S., Abouelregal A.E., 2013, The effect of dual-phase-lag model on reflection of thermoelastic waves in a solid half space with variable material properties, Acta Mechanica Solida Sinica, 26, 659-670 